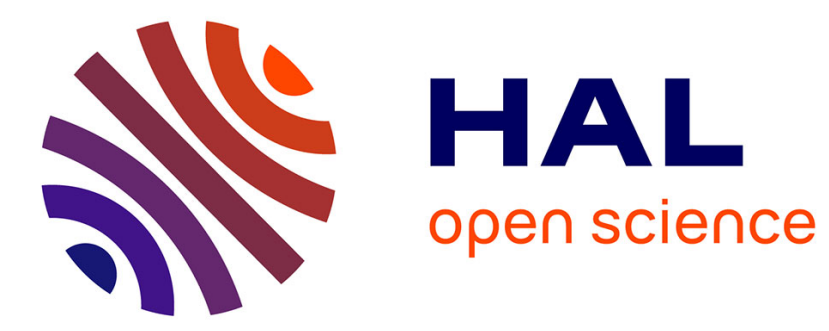

\title{
MOLECULAR DYNAMICS STUDY OF AMORPHIZATION INDUCED BY CHEMICAL DISORDER IN CRYSTALLINE NiZr2
}

C. Massobrio

\section{- To cite this version:}

C. Massobrio. MOLECULAR DYNAMICS STUDY OF AMORPHIZATION INDUCED BY CHEMICAL DISORDER IN CRYSTALLINE NiZr2. Journal de Physique Colloques, 1990, 51 (C4), pp.C455-C4-61. 10.1051/jphyscol:1990406 . jpa-00230766

\section{HAL Id: jpa-00230766 https://hal.science/jpa-00230766}

Submitted on 1 Jan 1990

HAL is a multi-disciplinary open access archive for the deposit and dissemination of scientific research documents, whether they are published or not. The documents may come from teaching and research institutions in France or abroad, or from public or private research centers.
L'archive ouverte pluridisciplinaire HAL, est destinée au dépôt et à la diffusion de documents scientifiques de niveau recherche, publiés ou non, émanant des établissements d'enseignement et de recherche français ou étrangers, des laboratoires publics ou privés. 


\section{MOLECULAR DYNAMICS STUDY OF AMORPHIZATION INDUCED BY CHEMICAL DISORDER IN CRYSTALLINE NIZr2}

\section{MASSOBRIO}

Composés non stoechiometriques, CNRS, Université de Paris Sud, Centre d'orsay, F-91405 Orsay Cedex, France

Résumé- Nous avons étudié par simulation en dynamique moléculaire la transformation de l'état crystallin à l'état amorphe dans l'alliage intermétallique NiZr2 qui se produit comme résultat de l'introduction de défauts antisites. Le comportement des fonctions de distribution de paires $g(r)$ montre que l'amorphisation a lieu pour des valeurs du paramètre d'ordre à longue distance $\mathrm{S} \leq 0.6$. La dilatation du volume qui en résulte est de l'ordre de $2 \%$.

Abstract - By means of constant temperature-constant pressure molecular dynamics we investigate the crystal-to-amorphous transformation in the intermetallic alloy $\mathrm{NiZr}_{2}$ resulting from the introduction of antisite defects. The behavior of the pair distribution functions $g(r)$ reveals that amorphization takes place for values of the long range order parameter $S \leq 0.6, t h e$ corresponding volume expansion being of the order of $2 \%$.

\section{1) INTRODUCTION}

A large number of intermetallic alloys can become amorphous when irradiated and destabilized at relatively low temperature depending on the system and the nature of the incident particle(1). A convincing experimental evidence supporting chemical disorder as the leader driving force in this kind of crystal-to-amorphous (C-A) transformation is given by the Brillouin scattering and transmission electron microscopy studies of softening,disordering and amorphization in $\mathrm{Zr}_{3} \mathrm{Al}$ performed by Okamoto,Rehn and coworkers in Argonne(2). This group established a close relationship between the critical dose of irradiation, the expansion of the lattice parameter $(\Delta \mathrm{V} / \mathrm{V}=2 \%)$ and the onset of amorphization corresponding to a threshold value $S=0.2$ of the long range order parameter. Moreover Okamoto et al. conjectured that the volume dependence of the shear elastic constant associated with radiationinduced chemical disordering and eventual amorphization is essentially identical to that associated with the heating to melting of many solids. Given the above context we set out to study by molecular dynamics (MD) simulations the influence of substitutional chemical disorder on the stability of crystalline $\mathrm{NiZr}_{2} \cdot \mathrm{NiZr}_{2}$ is particularly suited to our purposes because this material becomes amorphous not only by mechanical alloying(3) but also by electron irradiation(4). To allow for a legitimate comparison with experiments we exercised a great care to set up a realistic $\mathrm{N}$-body potential for $\mathrm{NiZr}_{2}{ }^{(5)}$, fitted to the alloy cohesive energy and elastic constants. To this purpose we related to a recent work on fcc transition metals (6) showing that a simple semi-empirical tight-binding scheme based on the second moment approximation to the electronic density of states can provide a good description of bulk and defects properties at zero temperature. Our simulations consisted of two steps: first we established the validity of the interatomic potential at high temperatures, then we create in our lattice the microscopic conditions corresponding to chemical disorder. To this end we selected one configuration from the constant-temperature constant- 
pressure molecular dynamics trajectories for the system at equilibrium at $\mathrm{T}=300 \mathrm{~K}$ and we simply exchanged a given number of $\mathrm{Ni}$ and $\mathrm{Zr}$ particles. The degree of chemical disorder is quantified in terms of the value taken by the long range order parameter $S$. The most practical definition of $S$ for our purposes is given by $S=(p-r) /(1-r)$ where $p$ and $r$ are respectively the probability of presence of an $A$-type atom $(A=N i$ or $Z r)$ on an ordered lattice site and the molar ratio of $A$ atoms. By monitoring the pair distribution functions upon dynamical relaxation we witnessed the crystal-toamorphous transformation for values of the long range order parameter $S \leq 0.6(5)$. On the other hand the crystal is stable against introduction of chemical disorder when $S>0.6$. Furthermore the amorphous pair distribution functions obtained by crystal destabilization are essentially identical to those resulting from a rapid quenching from the liquid state.

\section{2) MODEL AND COMPUTATIONS}

The crystalline structure of $\mathrm{NiZr}_{2}$ is of the $\mathrm{CuAl}_{2}(\mathrm{C} 16)$ type.We studied systems of two different sizes: $108 \mathrm{Ni}$ and $216 \mathrm{Zr}$ atoms $(3 \times 3 \times 3$ lattice cells forming a parallelepipedic box) as well as $256 \mathrm{Ni}$ and $512 \mathrm{Zr}$ atoms ( $4 \times 4 \times 4$ lattice cells). The expression for the cohesive energy reads

$$
E=\sum_{\alpha} \sum_{i_{\alpha}=1}^{N_{\alpha}}\left\{\sum_{\substack{\beta \\ i_{\alpha} \neq j_{\beta}=1}} \sum_{j \beta}^{N_{\beta}} A_{\alpha \beta} \exp \left[-p_{\alpha \beta}\left(\frac{r_{i j}^{\alpha \beta}}{d_{\alpha \beta}}-1\right)\right]-\sqrt{\sum_{\substack{\beta \\ i_{\alpha} \neq j_{\beta}}} \sum_{j \beta=1}^{N_{\beta}} \xi_{\alpha \beta}^{2} \exp \left[-2 q_{\alpha \beta}\left(\frac{r_{i j}^{\alpha \beta}}{d_{\alpha \beta}}-1\right)\right]}\right\}
$$

where $r_{i j} \alpha \beta=/ r_{i} \alpha-r_{j} /$ and the indexes $i_{\alpha}\left(j_{\beta}\right)$ run over all the particles. Interactions have been computed for distances $r_{i j} \alpha \beta$ within a spherical cutoff radius $r_{c}=0.53 \mathrm{~nm}$. The fitting procedure we employed is based on a set of potential parameters giving a remarkable agreement between experimental and calculated elastic constants. For more details on this aspect of our work we refer the reader to ref.5 and to an outcoming article (C.Massobrio,V.Pontikis,G.Martin, Phys.Rev.B to be published). Our molecular dynamics simulations are performed within the Nose-Andersen constant temperature,constant pressure (NPT) technique(7) and the equations of motion are solved via a fifth order predictor-corrector algorithm with timestep $\Delta t=10-15$ s. Periodic boundary conditions are applied in the three directions.

To study the reaction of our system to substitutional chemical disorder we introduced antisite defects in one configuration pertaining to a well equilibrated trajectory at $\mathrm{T}=300 \mathrm{~K}$. The long range order parameter $\mathrm{S}$ is equal to 1 . for the chemically ordered crystalline structure and to zero when $p$ equals the molar ratio. It is simple to impose a certain degree of chemical disorder by keeping a $\mathrm{Ni}$ atom on its position in the crystal with a probability given by $p=r+S(1-r)$. First we generate a sequence of $N$ random numbers $v_{n}$ in between 0 and 1 ( $N$ being the number of nickel atoms in the system). Next the position of the $n^{\text {th }}$ nickel atom is exchanged with the position of a randomly chosen zirconium atom provided $v_{n}$ is larger than the input probability $p$. Then the system is allowed to evolve under the NPT dynamics for up to $10^{5}$ time steps $\left(10^{-10} \mathrm{~s}.\right)$

\section{3) EOUILIBRIUM PROPERTIES}

We performed a series of simulations to establish the phase diagram of crystalline $\mathrm{NiZr}_{2}$ at zero pressure and test the dynamical behavior of our model. We recorded 
high diffusion values of the order of $\mathrm{D}=10^{-5} \mathrm{~cm} / \mathrm{s}^{2}$ at $\mathrm{T}=1535 \mathrm{~K}$ (the experimental melting point being equal to $T=1440 \mathrm{~K}$ ) for the system of $\mathrm{N}=768$ particles which melted after 200000 timesteps $\left(2 \cdot 10^{-10} \mathrm{~s}\right.$.). Therefore we can conclude that the melting point of our model is located at a temperature $T^{m e l} \leq 1535 \mathrm{~K}$. This estimate is consistent with the overheating that characterizes a model crystal in which extended defects are absent and the formation of point defects is prevented by the limited duration of the simulations. Further insight into the dynamical properties of our system can be gained by looking at the mean square displacement $\left\langle\mathrm{u}^{2}\right\rangle$ calculated along the $x$-direction. According to the Lindemann criterion the temperature of vibrational instability accompanying the melting of the crystal corresponds to a ratio $\mathrm{r}=\mathrm{u} / \mathrm{a}$ equal to 0.1 where $\mathrm{u}$ is the square root of the mean square displacement and a the nearest neighbor distance. In our case the mean square displacement at the experimental melting point is close to $0.06 \cdot 10^{-2} \mathrm{~nm}^{2}$ and the corresponding Lindemann ratio is $r=u / a \approx 0.08$ where in this case $a$ is the composition weighted average of nearest neighbors distances in the pure metals. On the basis of these results we conclude that the interatomic potential for $\mathrm{NiZr}_{2}$ we devised is well behaving when tested against the experimental thermodynamical properties of real $\mathrm{NiZr}_{2}$. In fact it performs remarkably well at high temperatures despite its being fitted to static properties at $T=0 \mathrm{~K}$ only. Finally we focused on the properties of the glass produced by rapid quenching of the melt. Starting from a configuration inherent to the melt at $\mathrm{T}=1600 \mathrm{~K}$ we cooled the system down to room temperature at a quenching rate equal to $4.2 \cdot 10^{13} \mathrm{~K} / \mathrm{s}$. At $\mathrm{T}=300 \mathrm{~K}$ we annealed the system for 60000 timesteps and we characterized the thermodynamic state by calculating the pair distribution functions $g(r)$. This step allows a direct comparison with the analogous results we obtain by crystal-to-amorphous transformations induced by chemical disorder.

\section{4) RESPONSE TO CHEMICAL DISORDER}

The introduction of chemical disorder has the primary effect of causing a volume increase of the system which relaxes to a stationary situation with negligeable changes in the values of thermodynamical and structural quantities throughout the length of our simulations $\left(10^{-10} \mathrm{~s}\right.$.). This effect is illustrated in Fig. 1 where we plot the average volume at $\mathrm{T}=300 \mathrm{~K}$ over a time interval that includes the equilibrium portion of the run and the phases of over expansion and relaxation of the crystal brought about by the presence of antisite defects. The values of the volume expansion $\Delta \mathrm{V} / \mathrm{V}_{\text {eq }}$ are quite similar for long range order parameters in between 0 and 0.7 (ranging from 0.024 to 0.016 ), less important is the expansion induced in the case $\mathrm{S}=0.9\left(\Delta \mathrm{V} / \mathrm{V}_{\mathrm{eq}}=0.007\right)$ corresponding to only $9 \mathrm{Ni}-\mathrm{Zr}$ exchanges for a system composed of $108 \mathrm{Ni}$ and $216 \mathrm{Zr}$ atoms. A microscopic insight into the response of the system is given by the temporal evolution of the mean square displacement $\left\langle\delta r^{2}\right\rangle_{t}$ calculated for $\mathrm{Ni}$ and $\mathrm{Zr}$ separately. The trend of $\left\langle\delta \mathrm{r}^{2}\right\rangle_{t}$ indicates that during the first 10000 steps the atoms seek a local rearrengement with an apparent diffusion coefficient of the order of $10^{-6} \mathrm{~cm}^{2} / \mathrm{sec}$. On the other hand the $\left\langle\delta \mathrm{r}^{2}\right\rangle_{\mathrm{t}}$ independently averaged over two subsequent runs of 10000 and 20000 steps each are limited to less than $0.1 \cdot 10^{-2} \mathrm{~nm}^{2}$. This result demonstrates that no long range diffusion of either atomic species detectable on the timescale of our simulations follows the introduction of chemical disorder.

Pair distribution functions $g(r)$ are the appropriate quantities to detect structural changes occurring in a crystalline structure. The transformation from the crystalline 
to the amorphous state is highlighted by the evident structural differences existing between the pair distribution functions (global and restricted to interactions between like $\mathrm{Ni}-\mathrm{Ni}$ atoms displayed in Fig.2 and in Fig.3) of $\mathrm{NiZr}_{2}$ computed before and after the imposition of the largest degree of chemical disorder. Also in Figs. 2,3 we plotted the $g(r)$ of the structure rapidly quenched. The disordered states obtained by chemical destabilization and rapid cooling from the liquid state (we denote them $\mathrm{AC}$ and AL) feature global g(r) nearly identical. On the other hand the $\mathrm{Ni}-\mathrm{Ni}$ pair distributions differ somewhat in shape,particularly in between 0.4 and $0.6 \mathrm{~nm} . \mathrm{By}$ taking the integral of the $\mathrm{Ni}-\mathrm{Ni} \mathrm{g}(\mathrm{r})$ up to the first minimum we obtain coordination numbers equal to 2 for the crystal,2.75 for $\mathrm{AC}$ and 3.72 for AL. These peculiarities suggest that the nickel atoms stow more compactly at short distances in the amorphous structure and particularly when it originates from the liquid state. For zirconium the coordination number decreases from 11 in the crystal to 10.5 in both amorphous structures. Therefore the rearrangement imposed by the chemical disorder affects more heavily the nickel than the zirconium atoms. Similar features were found in the experimental partial pair distribution functions obtained by neutron diffraction on samples of $\mathrm{Ni}_{36} \mathrm{Zr}_{64}$ prepared by rapid quenching from the liquid state. By Fourier transforming the partial interference functions $S(Q)$ Mizoguchi et al.(8) concluded that in amorphous $\mathrm{Ni}_{36} \mathrm{Zr}_{64}$ a nickel atom has an average of 3.3 nickel atoms nearest neighbors, while for zirconium the same quantity is equal to the value taken in the crystal.

By increasing $\mathrm{S}$ some signatures of the crystalline structure appear in the Ni-Ni pair distribution function beginning for $S=0.5$ and more clearly for $S=0.6$ and $S=0.7$ as shown in Fig.4. Negligeable changes are detected for $S=0.2$ and $S=0.4$. In Fig.4 relative to the $\mathrm{Ni}-\mathrm{Ni} \mathrm{g}(\mathrm{r})$ for $\mathrm{S}=0.6$ and $\mathrm{S}=0.7$ one notices two peaks developing out of the second shell of neighbors, the first lower than the second as in the unperturbed lattice. At $S=0.6$ the first peak of the global $g(r)$ becomes for the first time more prominent than the one located immediately at its right. This feature is characteristic of the crystalline solid (Fig.5).We believe this signature can provide a simple criterion to mark the boundary between crystal and amorphous nature. The structures obtained for $S=0.7$ are even more crystal-like either regarded through the behavior of the global or $\mathrm{Ni}-\mathrm{Ni} \mathrm{g}(\mathrm{r})$ strongly resembling the analogous quantities calculated for the equilibrium structure.

\section{5) CONCLUSIONS}

The main purpose of this work was to investigate by molecular dynamics simulations the effects of the presence of antisite defects on the stability of crystalline $\mathrm{NiZr}_{2}$. Our results prove unambigously that a moderate degree of chemical disorder is sufficient to induce the crystal-to-amorphous transformation. Already for $S=0.7$ the shape of the global pair distribution function differs radically from the one relative to the crystal. The volume expansion associated with amorphization is of the order of $2 \%$,it decreases slowly going from $S=0$ to $S=0.7$ and becomes less than $1 \%$ for a degree of disorder $(S=0.9)$ that does not destabilize the crystal. The results we obtained and the experimental data on $\mathrm{Zr}_{3} \mathrm{Al}$ lead support to the conjecture that a value of $\Delta \mathrm{V} / \mathrm{V}_{\mathrm{eq}}$ not too different from $2 \%$ might represent a universal threshold of instability beyond which an intermetallic alloy loses its topological order. 


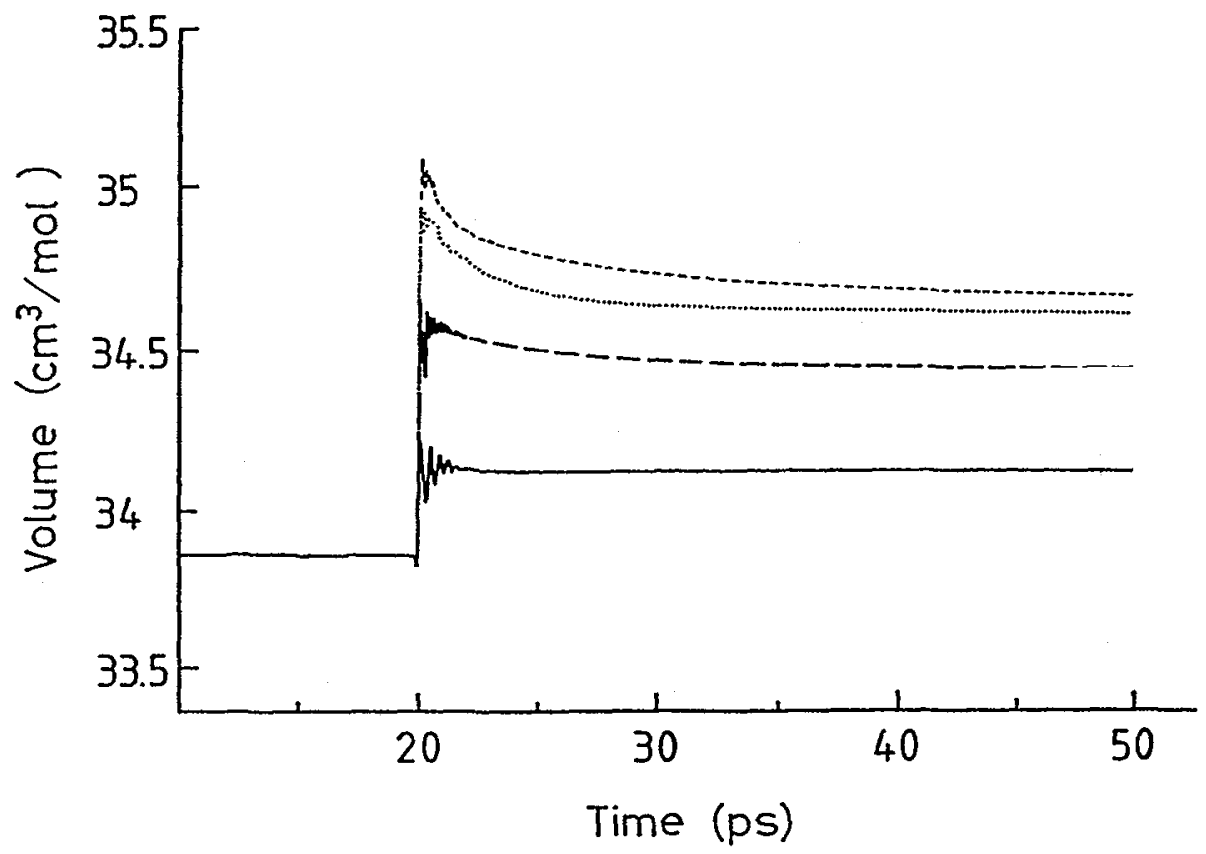

Fig.1 - Average volume of the system at $\mathrm{T}=300 \mathrm{~K}$ before and after introduction of chemical disorder.

Full line: $S=0.9$, dashed line $S=0.6$, dotted line $S=0.2$, short-dashed line $: S=0$

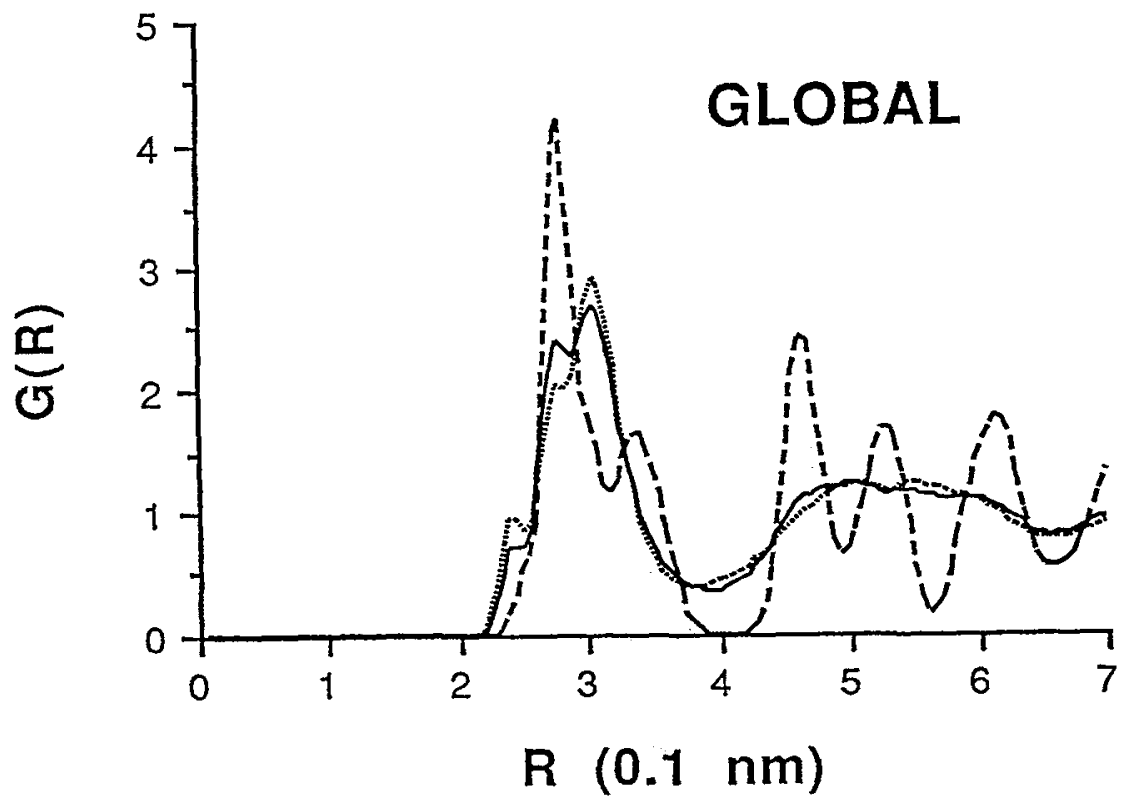

Fig.2 - Global pair distribution function at $\mathrm{T}=300 \mathrm{~K}$.Dashed line: crystalline NiZr2.Full line:after the imposition of chemical disorder, $S=0$. Dotted line:amorphous obtained by rapid quench from the liquid state. 


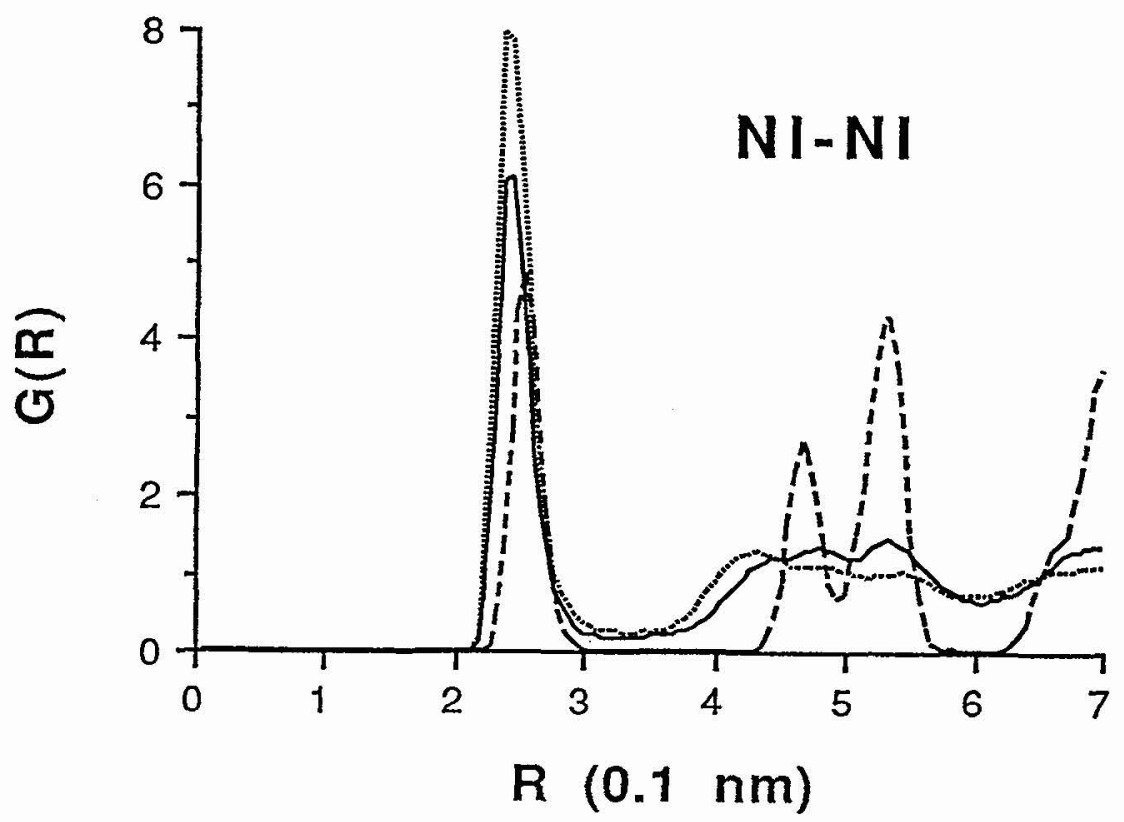

Fig.3 - Ni-Ni pair distribution function at $\mathrm{T}=300 \mathrm{~K}$.Dashed line: crystalline NiZr2.Full line:after the imposition of chemical disorder, $S=0$. Dotted line:amorphous obtained by rapid quench from the liquid state.

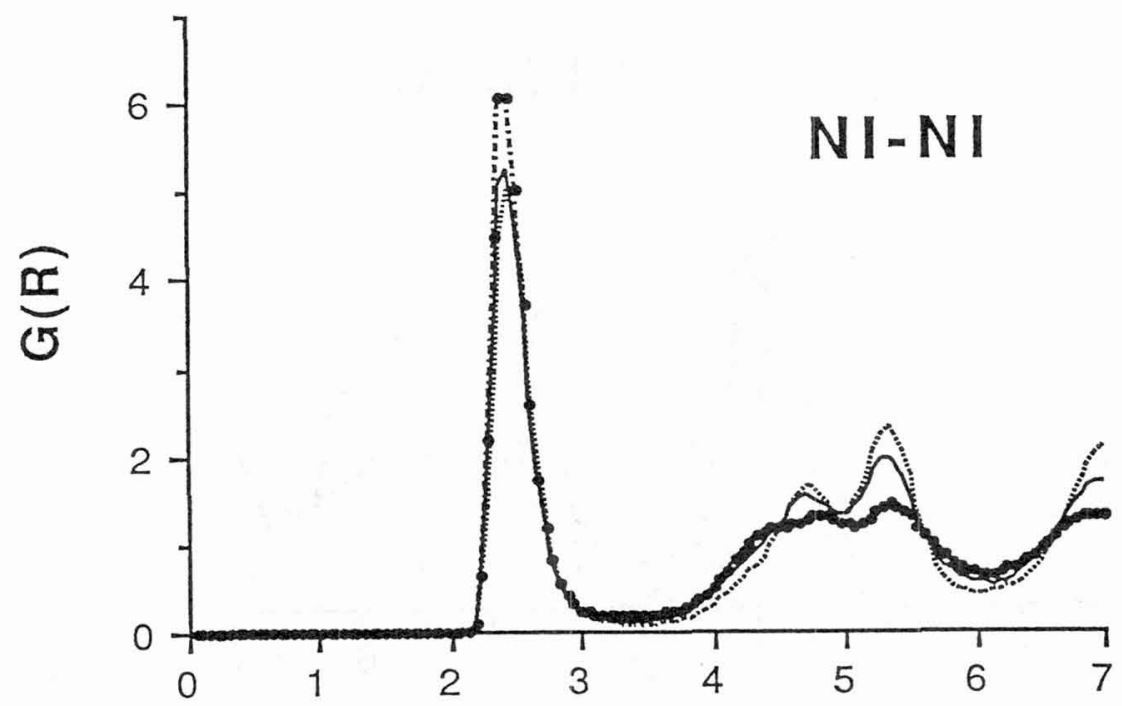

Fig.4 - Ni-Ni pair distribution function for three different degrees of chemical disorder at $\mathrm{T}=300 \mathrm{~K}$.

Dash-dotted thick line: $S=0$. Full line: $S=0.6$.Dotted line: $S=0.7$ 


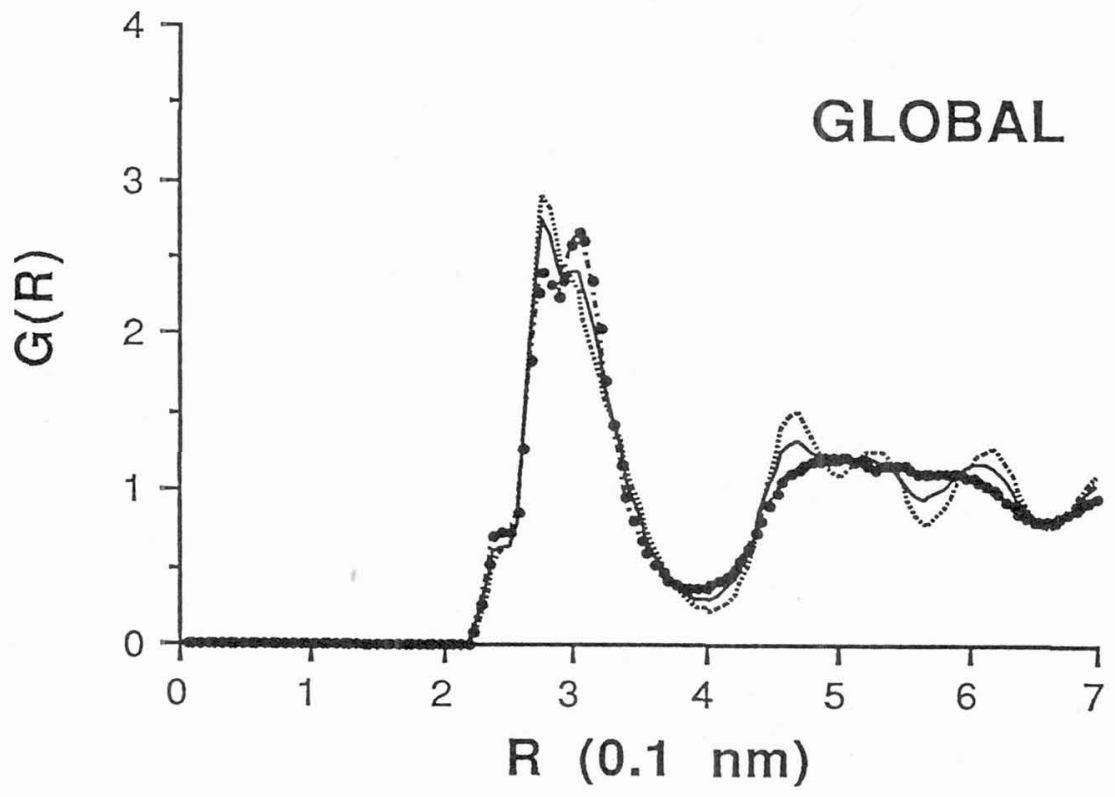

Fig. 5 - Global pair distribution function for three different degrees of chemical disorder at $T=300 \mathrm{~K}$.Dash-dotted thick line: $S=0$.Full line: $S=0.6$.Dotted line: $S=0.7$

\section{ACKNOWLEDGEMENTS}

The author of this article is grateful to Dr.G.Martin and Dr.V.Pontikis (SRMP,CEA Saclay) for their encouragement and support. C.M. also benefited from useful discussions with F.Willaime and P.Bellon.

\section{REFERENCES}

/1/ R.B.Schwarz,W,L.Johnson J.of the Less Common Metals 140,1(1988)

12/ L.E.Rehn,P.Okamoto,J.Pearson,R.Bhadra,M.Grimsditch Phys.Rev.Lett.59,2987(1987)

13/ E.Gaffet Mat.Science and Eng. A119,185(1989)

14/ H.Mori,H.Fujita,M.Tendo,M.Fujita Scr.Metall.18,783(1984)

15/ C.Massobrio,V.Pontikis,G.Martin Phys.Rev.Lett. 62,1142(1989)

16/ V.Rosato,M.Guillope,B.Legrand Phil.Mag.A 59,321(1989)

17/ C.Massobrio,V.Pontikis,G.Ciccotti Phys.Rev.B 39,2640(1989)

18/ T.Mizoguchi,S.Yoda,N.Akutsu,S.Yamada,J.Nishioka,T.Suemasa,N.Watanabe in $5^{t h}$ Int.Conf.on Rapidly Quenched Metals, Eds.F.Steeb and Warlimont (Wurtzbourg,Germany,Sept. 3-7,1984) North Holland p.483 (1985) 